\section{REVISTA}

Actualidades Investigativas en Educación

\title{
ESTUDIO DE FRACCIONES EN CONTEXTOS SONOROS
}

STUDY OF FRACTIONS IN SOUND CONTEXTS

\section{Volumen 16, Número 2}

Mayo-Agosto

pp. $1-21$

Este número se publicó el $1^{\circ}$ de mayo de 2016

DOI: http://dx.doi.org/10.15517/aie.v16i2.23933

\author{
Alexander Conde \\ Sandra Parada \\ Vicente Liern
}

Revista indizada en REDALYC, SCIELO

Revista distribuida en las bases de datos:

LATINDEX, DOAJ, E-REVIST@S, IRESIE, CLASE, DIALNET, SHERPA/ROMEO, QUALIS, MIAR

Revista registrada en los directorios:

ULRICH'S, REDIE, RINACE, OEI, MAESTROTECA, PREAL, CLACSO

Los contenidos de este artículo están bajo una licencia Creative Commons 


\title{
ESTUDIO DE FRACCIONES EN CONTEXTOS SONOROS
}

\author{
STUDY OF FRACTIONS IN SOUND CONTEXTS
}

\section{Alexander Conde \\ Sandra Parada ${ }^{2}$ \\ Vicente Liern ${ }^{3}$}

\begin{abstract}
Resumen: En este ensayo resaltamos los vínculos cognitivos entre las matemáticas y la música, que pueden favorecer procesos de enseñanza y aprendizaje de las fracciones en la matemática escolar. Las actividades exhibidas provienen de una investigación que promueve experiencias sensoriales en el campo rítmico para favorecer la construcción de nociones matemáticas. Dichas actividades posibilitan un engranaje armónico entre las matemáticas y la música convergentes en el tiempo y el sonido como los objetos de estudio común entre éstas disciplinas. El análisis de la investigación que aquí reportamos es producto de la aplicación de las actividades en diferentes programas de formación de profesorado de México y Francia, alrededor de la enseñanza y aprendizaje de las matemáticas en contextos interdisciplinarios. Este análisis se desarrolló desde tres categorías referentes a las nociones de unidad relativa, relación parte-parte y equipartición, las cuales son nociones fundamentales para el estudio de las fracciones en la matemática escolar. Encontramos que la enseñanza con enfoque integrador requiere no solo conocimientos especializados, sino un cambio de creencias en torno a las opiniones del profesorado sobre la organización del currículo, su enseñanza y la manera en que aprende la población estudiantil. Un aporte de esta experiencia es ofrecer a docentes elementos teóricos y didácticos para el estudio de las fracciones en contextos interdisciplinarios.
\end{abstract}

Palabras clave: FRACCIONES, SONIDO, TIEMPO, INTERDISCIPLINARIEDAD, FORMACIÓN DE PROFESORES.

Abstract: In this essay we highlight the cognitive links between mathematics and music, which can facilitate teaching and learning processes of fractions in school mathematics. The displayed activities come from a research that promotes sensory experiences in the rhythmic field to favor the construction of mathematical notions. These activities enable a harmonic gear between mathematics and music converging in time and sound as common objects of study between these disciplines. The analysis of the research reported here is the result of the implementation of activities in different teachers' training programs of Mexico and France, related to teaching and learning of mathematics in interdisciplinary contexts. This analysis was developed from three categories concerning the notions of relative unit, part-part relationship, and equipartition, which are fundamental notions for the study of fractions in school mathematics. We found that teaching with unifying approach requires not only specialized knowledge, but a change of beliefs about teachers' opinions on the organization of the curriculum, its teaching and the way in which students learn. A contribution of this experience is providing teachers theoretical and didactic elements for the study of fractions in interdisciplinary contexts.

Keywords: FRACTIONS, SOUND, TIME, INTERDISCIPLINARITY, TEACHER TRAINING.

\footnotetext{
1 Profesor-Investigador del Departamento de Ciencias Básicas de la Universidad de Medellín, Colombia. Doctor en Ciencias en la especialidad de Matemática Educativa. Dirección electrónica: Iconde@udem.edu.co

2 Profesora-Investigadora de la Escuela de Matemáticas de la Universidad Industrial de Santander, Bucaramanga-Colombia. Doctora en Ciencias en la especialidad de Matemática Educativa. Dirección electrónica: sparada@matematicas.uis.edu.co

${ }^{3}$ Catedrático del Departamento de Matemáticas para la Empresa y para la Economía de la Universidad de Valencia, España. Doctor en Física Teórica. Académico numerario de la Real Academia de Doctores. Académico numerario de la Real Academia de Ciencias Económicas y Financieras. Dirección electrónica: vicente.liern@uv.es
}

Ensayo recibido: 14 de mayo, 2015

Enviado a corrección: 28 de setiembre, 2015

Aprobado: 7 de marzo, 2016 


\section{Introducción}

Los trabajos realizados en educación matemática que han intentado aproximar la música con las matemáticas han usado elementos musicales como herramientas motivacionales hacia los objetos matemáticos. No obstante, desde nuestra perspectiva, dichas aproximaciones han hecho poco hincapié en dos elementos esenciales de la música: el tiempo y el sonido. Estos componentes, por separado y analizando sus relaciones, vislumbran un amplio campo de estudio de la física y las matemáticas.

Los objetos físicos, musicales y matemáticos pueden presentarse en el aula mediante artefactos computacionales que permiten representaciones dinámicas y posibilitan un engranaje interdisciplinario entre las áreas involucradas. En este sentido, consideramos el tiempo y el sonido como los objetos de estudio común donde se engrana una relación armónica entre las matemáticas, la física y la música (Conde, 2013).

En investigaciones de Naik y Subramaniam (2008) y Prediger y Schink (2009) se reporta que uno de los temas de la matemática escolar al que el estudiantado se enfrenta con mayor dificultad es el aprendizaje de las fracciones. Con la intención de aportar estrategias de enseñanza alternativas que ayuden a superar dicha problemática, valoramos en los elementos rítmicos una posibilidad de acercamiento al estudio de las fracciones.

El ritmo, en el contexto de la música, está determinado por la duración de los sonidos y silencios. Las figuras y silencios musicales permiten manejar el tiempo como un ente discreto a través de intervalos de duración de sonidos, o ausencia del mismo. El valor relativo de las figuras musicales, establece un orden estricto: cada figura dura la mitad de la anterior y el doble de la siguiente. Esta relación construye un sistema de signos rítmicos musicales que surge a partir de su unidad de medida y cuya estructura se fundamenta en las fracciones.

Consideramos que las experiencias del alumnado en el espacio-tiempo musical pueden dar significado a las nociones de unidad relativa, relación parte-parte y equipartición, que resultan imprescindibles para la construcción de la noción de fracción.

\section{Vínculos entre matemáticas y música}

Los vínculos entre las matemáticas y la música se inician formalmente en el mundo occidental con el estudio de la naturaleza de los sonidos musicales hallados por Pitágoras (570-479 a.C.). Parece ser que a través de la experimentación encontró relaciones entre la frecuencia y la longitud de cada porción de la cuerda sometida a vibración. El legado científico-tecnológico pitagórico se convirtió en un punto de referencia para quienes se 
dedican a la ciencia y el arte en la búsqueda del desarrollo de la teoría musical y sus componentes, como son: la armonía, la melodía, el timbre y el ritmo, principalmente.

En este trabajo aceptaremos que el ritmo musical se caracteriza como el evento sonoro constituido por la repetición de un patrón de sonidos y silencios organizado en una línea de tiempo (Conde, 2009). Este patrón está medido por el pulso y determinado por acentos. Este pulso o pulsación, que es la unidad básica de tiempo en música, se obtiene a partir de un evento que se repite regularmente y permite conocer si un sonido es largo o corto. En otras palabras, representa el latido de la música en cada obra; al igual que el del corazón, se mide por medio de número de pulsaciones por minuto. Para medir la duración de un sonido o ausencia del mismo, se hace necesario utilizar un sistema de representación que permita establecer relaciones entre diferentes tiempos de duración.

Conviene señalar que los vínculos matemático-musicales van más allá de su desarrollo histórico conceptual, de fórmulas y patrones matemáticos presentes en la composición musical y en la propia naturaleza de los sonidos, donde innegablemente se vincula el estudio de la acústica al campo de la física. En este sentido, advertimos la presencia de conexiones cognitivas entre las matemáticas y la música, tal y como señala Gardner (1997), quien establece enlaces entre las capacidades musicales y ciertas capacidades espaciales considerando que puede ser la música un organizador privilegiado de los procesos cognitivos. También Rauscher, Shaw, Levine, Wright, Dennis y Newcomb (1997) mencionan que "tocar una melodía implica la reconstrucción de un modelo espacial-temporal en el que las piezas del rompecabezas, se deben acoplar teniendo en cuenta las notas altas y bajas así como las de larga y corta duración" (p. 31). Estos estudios nos permiten afirmar que la música está ligada al aprendizaje cognitivo, en particular en el ámbito de la lógica, el razonamiento espacial, y el razonamiento abstracto. Además, nos permite ahondar en el espacio-temporal y su vínculo con los números fraccionarios en el sentido Rauscher y Zupan (2000), quienes afirman que la enseñanza musical mejora el razonamiento proporcional en relación con ciertos conceptos matemáticos, como la comprensión de las fracciones. Asimismo, confirma la presencia de estructuras espacio-temporales en el razonamiento de varias operaciones matemáticas y específicamente destaca la importancia en la comprensión de las proporciones y fracciones. Vaughn (2000) afirma que esta comprensión se constituye en un pre-requisito para el aprendizaje de las matemáticas en los niveles superiores.

Desde estudios de Conde (2013) respecto a estructuras espacio-temporales, encontramos que en la composición de los patrones rítmicos en el espacio-temporal musical 
puede ser conectada la idea de partición con la noción de fracción, a través de significados y usos de la unidad relativa. Bajo esta perspectiva, se pudo construir una trayectoria cognitiva de procesos que van esculpiendo un sendero por donde viaja la noción de tiempo y su unidad relativa. En conclusión, a la trayectoria cognitiva de la noción de tiempo y su unidad relativa la concebimos como la configuración de tres dimensiones. Configuración determinada por las situaciones y movimientos articulados entre las dimensiones de: niveles cognitivos, procesos observables y momentos de tránsito ${ }^{4}$. En la Figura 1, apreciamos las relaciones entre las caras, en ellas observamos que los momentos de percibir el tiempo, variaciones del tiempo de duración y sistema de medida del tiempo están presentes desde el nivel de identificación hasta el nivel de significación.

Los momentos de unidad relativa y construcción y ejecución de patrones rítmicos surgen desde el nivel de categorización, mientras que los momentos de adición y multiplicación, relación de equivalencia y equipartición surgen en el nivel de Asociación. El orden jerárquico de los momentos hacen imprescindibles la construcción de los primero para la construcción de los últimos.

En la cara lateral del cubo se encuentran los procesos observables que nos muestran la interpretación, representación y usos de la noción de tiempo y su medida tanto en los niveles como en los momentos.

Figura 1.

Trayectoria de la noción de tiempo y su unidad relativa

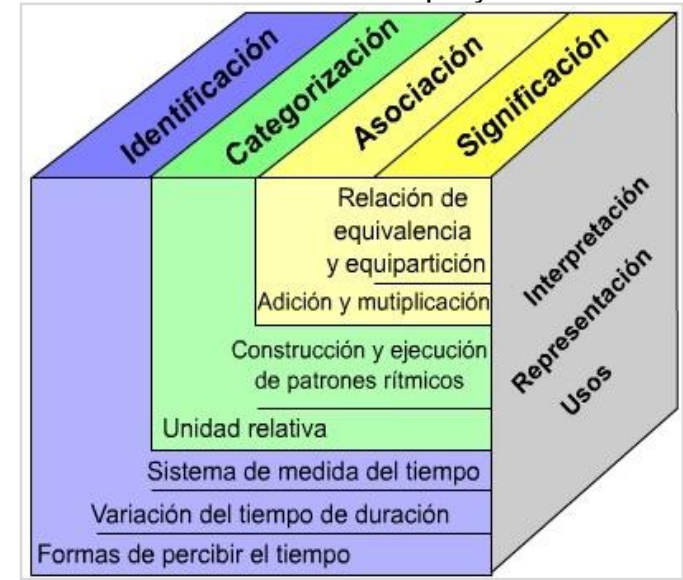

Fuente: Las unidad relativa como vínculo cognitivo entre el tiempo musical y las fracciones (Conde, 2013, p. 135).

\footnotetext{
${ }^{4}$ En este trabajo concebimos momentos de tránsito como las etapas experimentadas por el estudiantado donde se implica la noción de tiempo y su unidad relativa, estos son: formas de percibir el tiempo, variaciones del tiempo de duración, sistema de medida del tiempo, unidad relativa, construcción y ejecución de patrones rítmicos, adición y multiplicación, relación de equivalencia y equipartición.
} 
Por lo tanto, la construcción de la noción de tiempo y su unidad relativa en el estudiantado circula desde su percepción como estímulo inicial y refinamiento de la misma hasta sus significados y usos. Tal circulación es mediada por experiencias sensoriales apoyadas en las interacciones entre nuestra población estudiantil con percepciones y representaciones de objetos matemático-musicales.

Las investigaciones antes mencionadas y nuestra propia experiencia nos inducen a pensar que el contexto musical puede convertirse en una herramienta cognitiva para el aprendizaje de las fracciones con base en su estructura métrica. Por estas razones, vislumbramos grandes posibilidades motivadoras y posibles acercamientos didácticos para el docente que puede promover experiencias significativas alrededor de las fracciones desde el ambiente musical.

\section{Aspectos conceptuales y teóricos}

Para el desarrollo de la actividad musical, la necesidad de crear un sistema de signos que mostrara los valores relativos de las notas dentro de una única línea melódica era inevitable. Según Tiburcio (2002) a partir del siglo IX se fue consolidando la necesidad de una notación rítmicamente exacta como la notación proporcional. En el advenimiento del Ars nova en el siglo XIV, se introduce una notación similar a la actual, constituida por una sucesión de figuras representativas de diferentes duraciones: máxima, longa (cuadrada), breve (redonda), semibreve (blanca), mínima (negra), semimínima (corchea), fusa (semicorchea), semifusa (fusa). La longa se dividía en 2 ó 3 breves (según indicación específica), y el resto se dividía en 2 para pasar a la siguiente.

La trascendencia de la propiedad: que de cada figura es la mitad que la anterior y el doble que la siguiente, es utilizada para la construcción de las figuras y silencios musicales (ver Figura 2) como representaciones encargadas de medir el tiempo de duración de un sonido o ausencia del mismo. 
Figura 2.

Representaciones y valores de las figuras y silencios musicales

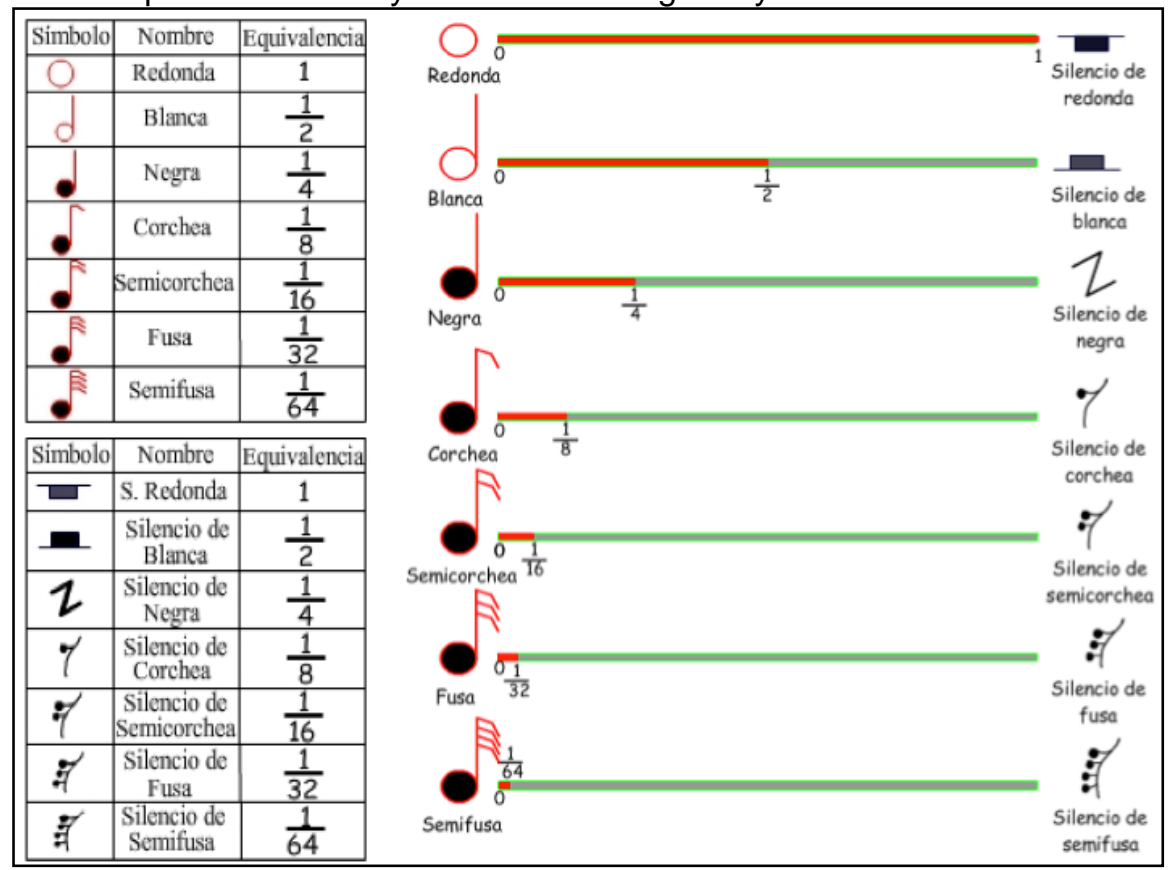

Fuente: Construcción y simulación propia.

Estas representaciones permiten fijar la música en el tiempo y escribir su experiencia temporal en dos dimensiones: el tiempo físico (segundos) y el tiempo musical (pulsos). Dentro del sistema de representación de figuras y silencios musicales se puede identificar la "unidad" determinada por el mayor tiempo de duración del sonido asociado a la figura redonda.

Cabe precisar que el valor en tiempo físico que se le asigna a la figura redonda depende de varios factores que se relacionan con la estructura métrica y la velocidad de la interpretación de una obra; por esta razón, a la unidad se le ha denominado "unidad relativa" en este escrito. En el sistema en mención, el papel de las fracciones es indispensable por dos motivos:

a) asignar un valor en términos del tiempo de duración de un sonido a cada figura,

b) permitir hacer comparaciones para crear relaciones de orden entre las figuras.

Es así como, desde nuestro estudio, concebimos la dimensión temporal como un campo propicio para estudiar la fracción como medida. Freudenthal (1983) considera que las representaciones con pares de objetos geométricos (como segmentos lineales, dominios planos), pueden ser presentados por derecho propio para dar cuerpo a fracciones, o pueden 
ser representativos de otras clases de pares de objetos como intervalos de tiempo, que han de ser entendidos en su razón fraccionaria. Por ello la propiedad de representar al tiempo de manera lineal, será aprovechada en este trabajo para formular las relaciones espacio y tiempo.

Por involucrar un fenómeno acústico, las formas de percibir -visual, auditiva y gestualestán mediadas por herramientas que le proporcionan argumentos de comprobación para dar cuenta, por sus propios medios, sobre la construcción y el significado de un concepto estudiados. Vygotsky (1988) señala que las herramientas son mediadoras semióticas de la actividad humana para la construcción de procesos psicológicos superiores. Estas herramientas pueden ser técnicas y psicológicas.

Los dos tipos de herramientas definidos por Vygotsky (1988) se pueden considerar respectivamente equivalentes a los conceptos de artefacto e instrumento definidos por Vérillon y Rabardel (1995). Estos autores consideran como artefacto al objeto material o abstracto (posiblemente sin significado para el usuario), destinado para realizar cierto tipo de actividad. Solamente con el uso del artefacto con un objetivo específico, el usuario le asigna significado y lo instaura como instrumento útil que media la actividad delimitada. Por lo tanto, el instrumento es considerado como constructo cognitivo, producto de una relación significativa entre el artefacto y su proceso de apropiación. "Es una entidad mixta, parte artefacto, parte esquemas cognitivos los cuales lo hacen un instrumento" (Artigue, 2002, p. 250).

Nuestra propuesta es plantear la construcción cognitiva de la unidad relativa como relación parte-parte y la equipartición de fracciones. Aquí identificamos dos tipos de artefactos: a) los artefactos de carácter simbólico, el sistema matemático de signos y el sistema musical de signos, y b) los artefactos computacionales, los cuales nos permiten desarrollar representaciones dinámicas virtuales y simulaciones de objetos musicales figuras y silencios musicales- y matemáticos -fracciones- como estructura para determinar el tiempo de duración de un sonido.

En este sentido, Trouche (2005) sostiene que la aparición de artefactos computacionales en la clase de matemáticas supone un problema de carácter didáctico acerca de transformar los artefactos, es decir, las computadoras o recursos tecnológicos en verdaderos instrumentos de actividad matemática y no como "recursos que resuelven y solucionan" problemas en el aprendizaje. Son artefactos que deben contribuir al desarrollo del pensamiento matemático buscando generar en su mente una reorganización conceptual. 


\section{Recurso didáctico para la enseñanza}

Actualmente, los profesores tienen acceso a multitud de recursos: objetos manipulables, artefactos digitales, medios virtuales, entre otros. No obstante, como lo mencionan Parada y Sacristán (2010) la incorporación improvisada, sin la planeación cuidadosa de la actividad matemática que se va a gestionar en el aula, puede desviar los objetivos de aprendizaje y generar confusión el estudiantado respecto a los objetos matemáticos de estudio. Contemplar recursos en clase implica cierto bagaje de conocimientos del profesor en torno a la conducción de su clase, a las maneras de usar los recursos que han seleccionado, de acuerdo a la actividad matemática que tiene prevista para el aula (Parada y Pluvinage, 2014). Estas son algunas reflexiones que inevitablemente el profesor hace sobre su práctica para discernir y pensar sobre cómo, cuándo y dónde usar un recurso, dependiendo de los propósitos de aprendizaje que deseen lograrse.

En experiencias con la formación de profesores alrededor de relaciones matemáticomusicales eventos realizados con profesores en diferentes espacios nos han permitido cimentar algunas reflexiones, como las Jornadas Formativas del Estado de México 2011; las Journées Mathématiques de l'IFÉ 2011 en Lyon (Francia); y el II Encuentro de Investigación e Innovación para la Formación Docente 2014, Hermosillo (México). Dichas reflexiones se constituyen en el objeto de divulgación de este ensayo.

Sin tener en cuenta las diferencias sociales y culturales de las personas docentes, se han realizado experiencias con ellas implementando actividades recuperadas del trabajo de Conde (2013); de este modo, se han podido evidenciar preocupaciones comunes del profesorado. Bajo esa perspectiva, quienes son docentes coinciden en los dominios conceptuales requeridos que implica el desarrollo en el aula de actividades matemáticas en el contexto musical. Vale la pena aclarar que la enseñanza con enfoque integrador entre las matemáticas y la música en el entorno escolar requiere, además, de conocimientos especializados, un cambio de creencias en torno a las opiniones de los profesores sobre la organización del currículo, su enseñanza y cómo aprenden las estudiantes y los estudiantes (Still y Bobis, 2005).

Sin embargo, el diseño de esta propuesta nos parece adecuada para el aula aunque el profesor o profesora no tenga conocimientos profundos en ninguna de las disciplinas vinculadas: física, matemáticas y música. Pensamos que sería más una decisión que enlaza las creencias y concepciones del profesor para implementar en sus clases nuevas formas de aproximación al estudio de las fracciones. La viabilidad de aplicación de la propuesta la 
sustentamos en los siguientes aspectos:

i) Contexto. Partimos de contextos reales que generan curiosidad y abren la posibilidad de indagar sobre las relaciones existentes entre las matemáticas y la música. En este sentido, Liern (2011) y Conde, Figueras, Pluvinage y Liern (2011) proponen las relaciones entre las fracciones y algunos elementos musicales como un tema interesante que le ofrece a docentes argumentos para una posible enseñanza del tema. Creemos que las experiencias rítmicas aportan un contexto real y próximo al alumnado en el que pueden encontrar significados de la noción de unidad relativa, parte-parte y equipartición, nociones implícitas en el tratamiento del tiempo y el sonido musical.

ii) Percepción natural. La música está compuesta por cuatro elementos esenciales: la melodía, la armonía, el timbre y el ritmo. En este caso, nos enfocamos en un solo elemento "el ritmo", por su carácter temporal, su independencia de los otros elementos y por su percepción natural. "La percepción del ritmo es natural para la mayoría de las personas, ya que desde los primeros años de vida se reconocen sonido y patrones rítmicos" (Shilling, 2002, p.180). De este modo, sin tener un contacto visual niñas y niños identifican a su padre por medio de sus pisadas, en las cuales determinan un patrón rítmico particular. Por lo general, las personas bailan de acuerdo al ritmo que escuchan, o al menos el que ellas perciben.

iii) Artefactos computacionales. Concebimos imposible lograr un acercamiento a nuestros propósitos sin el uso de artefactos computaciones que nos permitan entrelazar objetos matemático-musicales y darles cuerpo con diversas representaciones dinámicas y estáticas creadas por dichos artefactos computacionales. Para tal fin, creamos un ambiente computacional amigable tanto para docentes como para estudiantes. Consiste en una secuencia de simulaciones fácilmente manipulables que viene complementada con una hoja de trabajo. El ambiente está desarrollado en Flash Macromedia $\mathrm{CS}^{5}$, cuya película swf abre en cualquier explorador de Internet y puede ser trabajado online 0 descargado desde la página: http://alconde23.wix.com/alexander-conde

iv) Adaptación curricular. El acercamiento didáctico interdisciplinario que presentamos aquí, además de conectar nociones entre las matemáticas, la música y la física, también es una secuencia didáctica con la posibilidad de ser adaptada por el

${ }^{5}$ Lenguaje de programación orientado a objetos Adobe Flash Macromedia CS3 
profesorado a las necesidades de su aula de clase. Creemos que el trabajo de esta actividad también puede ser útil para docentes de música o ciencias naturales según los intereses y enfoques que deseen darle a la propuesta.

\section{Experiencias rítmicas en contextos de la matemática escolar}

Nuestro trabajo apunta a proveer experiencias diferentes al tradicional tratamiento de las fracciones en la matemática escolar (el pastel). En nuestro caso, partimos de un fenómeno real que a través de su estudio mediado por artefactos computacionales surgen conexiones entre las disciplinas. Las actividades aquí expuestas se retoman de una investigación más amplia cuyo proceso metodológico tiene una primera fase de diseño y desarrollo curricular porque construimos una propuesta didáctica interdisciplinaria. Y la segunda parte se ubica dentro de una perspectiva cualitativa de análisis, dado que nos interesa describir las formas utilizadas por nuestros alumnos y alumnas para manejar las nociones construidas sobre la unidad relativa, por medio de experiencias virtuales y reales que nos permitirán captar en las expresiones y representaciones del estudiantado, su manera de interpretar y usar los vínculos existentes entre las figuras musicales y las fracciones.

Desde dicha perspectiva interdisciplinar, en los siguientes párrafos ilustramos las actividades propuestas para el profesorado en el marco de las actividades de "tiempo y pulso" desde tres categorías referentes a las nociones de unidad relativa, relación parteparte y equipartición. Para tal fin, se exhibirá por categoría las imágenes originales de las representaciones dinámicas usadas en las actividades.

\subsection{Idea de unidad relativa}

Según Galperin y Georgiev (1969), cualquier conjunto puede también ser pensado como una unidad y, por lo tanto, la "unidad" en este caso adquiere otras designaciones numéricas distintas de uno. Esta aserción nos sugiere tener presente la relatividad de la unidad en términos de cantidad y de dimensión, ya que cambia según la nueva unidad establecida para realizar el proceso de medición.

El uso de la unidad relativa permite tratar el tiempo de forma discreta. Desde el punto de vista de Piaget (1978) la noción de tiempo es una de las más complejas en el aprendizaje de la población estudiantil de entre 8 y 12 años de edad. Según Piaget (citado por Sánchez, 1999) para la población infantil no hay una intuición primaria del tiempo de duración, sino que 
la evalúa a partir de la relación que establece entre una acción y la velocidad con la que se realiza. Al principio, la velocidad es una intuición: se intuye que alcanza un objeto o que le sobrepasa. Después, a medida que se va produciendo el estadio de las operaciones concretas, se concibe la velocidad como la relación entre el espacio recorrido y el tiempo empleado. O sea, la noción de tiempo se construye a partir de las nociones espaciales y de experimentaciones de distintas velocidades.

Para apreciar la duración del tiempo, en este trabajo se introduce el concepto de medida haciendo uso de la idea de espacio recorrido en un tiempo determinado mediante situaciones en las que el estudiantado mida con unidades convencionales (segundos y pulsos) y no convencionales (recubrimiento de barras, trazas) los intervalos cortos o largos de duración de tiempo de sonido. Las situaciones también permiten trabajar no sólo el tiempo medido de la duración de un sonido, sino lograr una estimación total del tiempo percibido.

El proceso de medición de tiempo exige la elección de una unidad, comparar esa unidad con una nueva magnitud y decir el número de unidades que "caben" en la magnitud dada. La comparación en nuestro caso se realiza por medio de artefactos computacionales que permiten introducir la noción de unidad relativa con el uso de representaciones audiovisuales simultáneas que articulan la medida de longitudes, recubrimiento de barras, tiempos de duración y número de pulsaciones (Figura 3).

Las experimentaciones de la unidad relativa en los dos espacios temporales producen en el estudiantado una sensación de variación en la estimación del tiempo. Las diferentes representaciones ofrecen simultáneamente la percepción de la duración de un sonido medido por el cronómetro (tiempo físico), el metrónomo ${ }^{6}$ (tiempo musical) y el recubrimiento de las barras. Los acercamientos y representaciones que la población estudiantil tiene con los objetos matemático-musicales caracterizan los procesos de concretización de dichos objetos.

\footnotetext{
${ }^{6}$ El metrónomo es un aparato que indica un tempo constante para regular la medida de los compases en las composiciones musicales.
} 
Figura 3.

Idea de unidad relativa

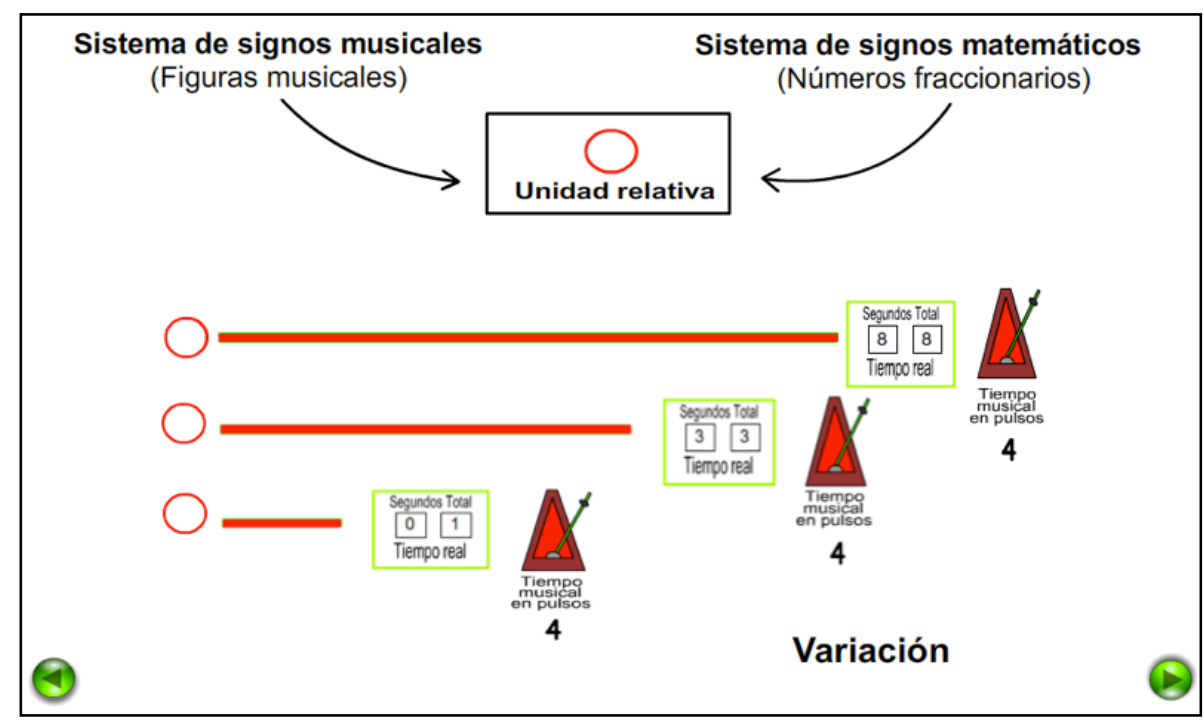

Fuente: Construcción y simulación propia.

\subsection{Relación parte-parte}

Continuando con la idea anterior del reconocimiento pleno de la unidad. Con la actividad que se presenta en la Figura 4, se pretende mostrar la unidad relativa con dos representaciones dinámicas: la pulsación percibida por el tic-tac de un metrónomo, generando al mismo tiempo un sonido y una traza sobre el metrónomo. Aquí el estudiante puede apreciar una medida de la unidad determinada por sus cuatro pulsos regulares y las dos diferentes trazas marcadas sobre el metrónomo.

Dotamos al estudiantado de una noción de unidad diferente de "uno" en concordancia con Galperin y Georgiev (1969); para dichos autores la rigidez y caracterización de la unidad -como único e individual asociado al trabajo con un solo tipo de unidad en situaciones de conteo y de medida- puede determinar el significado ambiguo de "unidad". Como parte de la solución de la situación anterior, Steffe, Cobb, Glasersfeld (1988) señalan que la variedad de tipos de la unidad y de partes de la unidad por medio de la experimentación proporcionan una fundamentación más adecuada para la comprensión de número entero; y esto supone un puente cognoscitivo para el aprendizaje de conceptos y operaciones de números racionales. 
Figura 4.

Medida de la unidad relativa

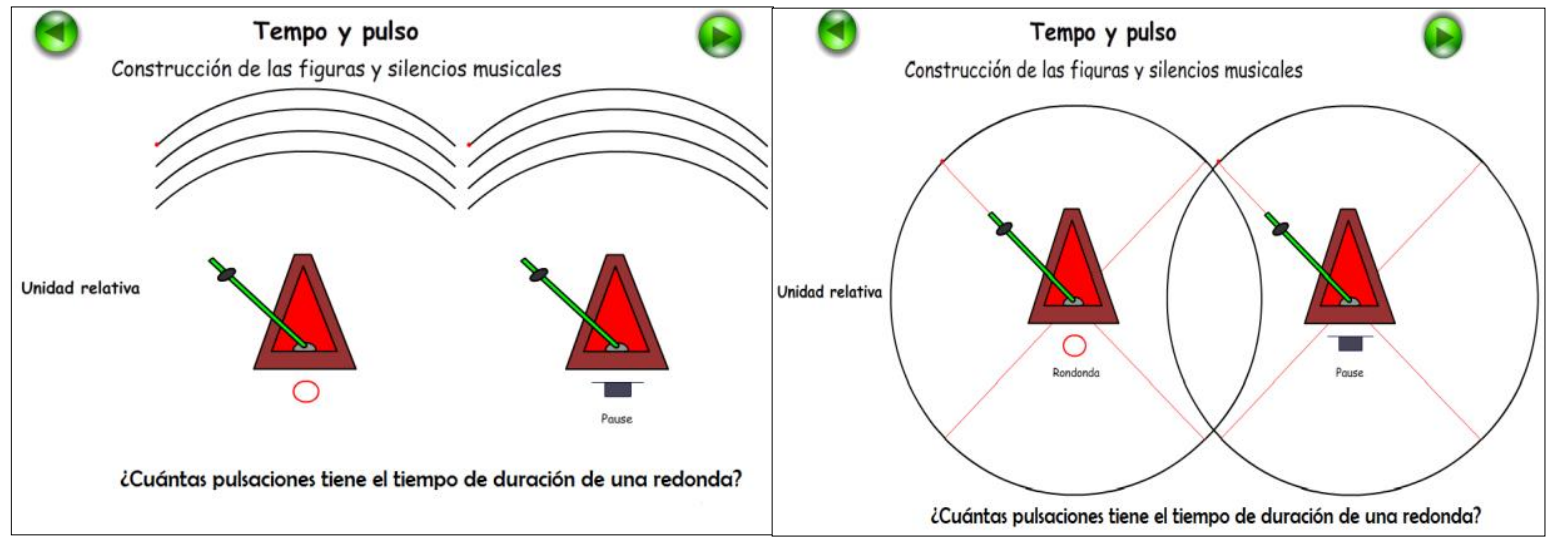

Fuente: Elaboración propia.

Varias investigaciones sobre fracciones han demostrado que muchos de los errores son cometidos por el estudiantado cuando se trabaja con ellas; dichos errores pueden ser una consecuencia de no entender que los números naturales y racionales implican ideas diferentes. Al respecto Kieren $(1976,1988)$ y Socas $(1997)$, mencionan que por ejemplo las y los estudiantes tienen la idea de que la "multiplicación hace más grande una cantidad", y al ver que los términos de una fracción cuando se multiplican por cierta cantidad de manera independiente se vuelven más grandes, confirman su suposición, pues no ven todavía a la fracción como un sólo número. Prediger y Schink (2009) señalan que un significado importante de la multiplicación de fracciones es la "parte de parte de un conjunto" (part-ofpart-model), porque $\frac{4}{5} \cdot \frac{2}{3}$ se interpreta como $\frac{4}{5} d e \frac{2}{3}$. La comprensión que la población estudiantil adquiere de este modelo es a menudo limitada por la dificultad de cambiar totalidades referentes o conjuntos.

En esta dirección queremos proporcionar situaciones donde construya en un contexto musical el significado de la relación parte-parte en las fracciones. La forma de hacer discreto el tiempo de duración del sonido por medio del conteo de la pulsación puede generar en ellos una sensación de partición en el campo temporal. Esto puede además favorecer la construcción de la noción unidad y la relatividad de la misma.

En el caso concreto, cuando aplicamos a la figura redonda (unidad relativa) la propiedad: cada figura es la mitad de la anterior y el doble de la siguiente, en el orden estricto en que se mencionan. Aquí se están formando parte de la unidad y parte de parte de la unidad como se observa en la Figura 5. 
En cada caso, las marcas de las trazas de la pulsación, relacionada a su expresión fraccionaria del tiempo musical de la figura o silencio, ayudan al estudiantado a establecer una sucesión.

Figura 5.

Parte de la unidad relativa y parte-parte

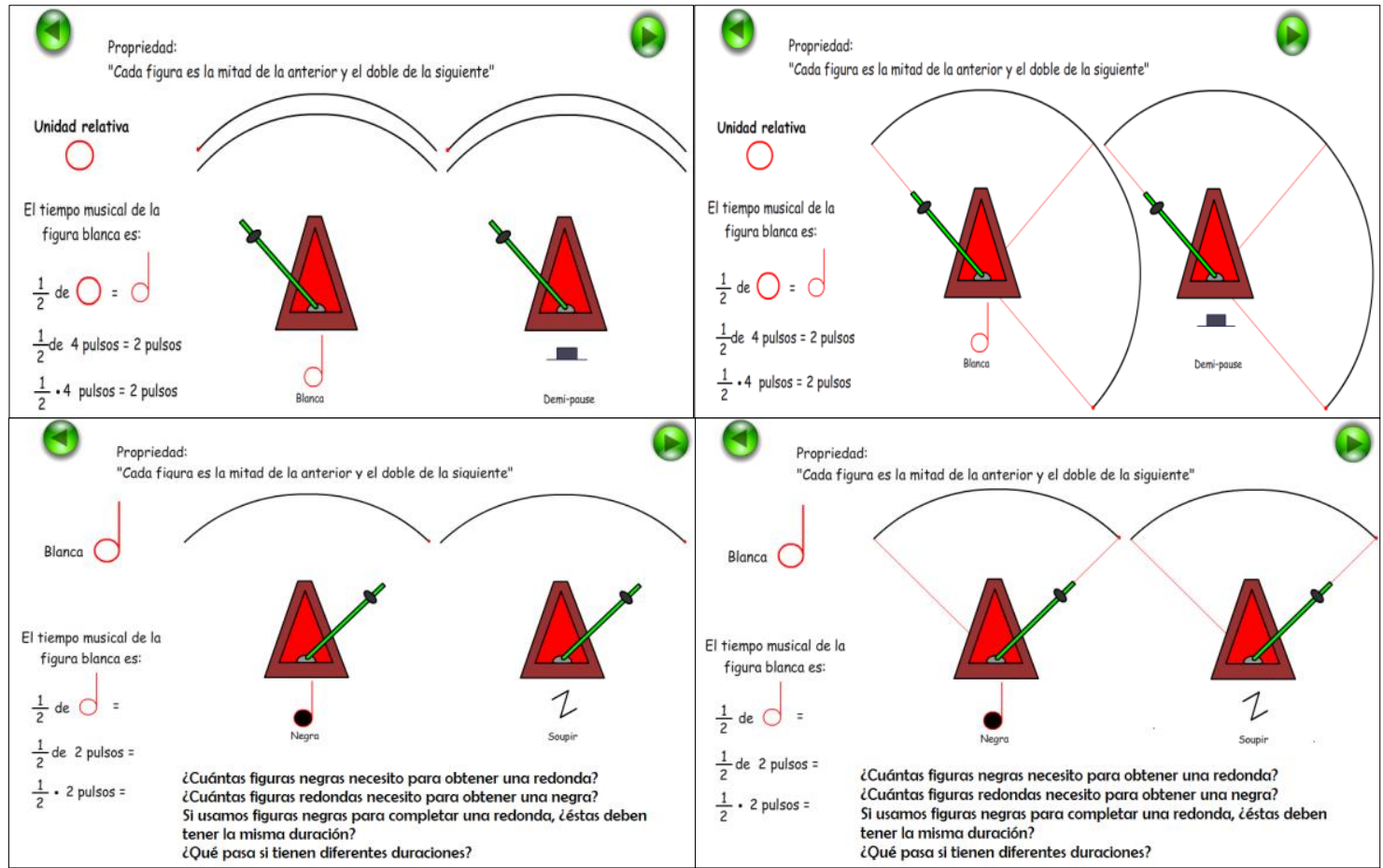

Fuente: Elaboración propia.

Como se observa en la Figura 5, los fraccionamientos de las figuras: redonda, blanca y negra, concuerdan exactamente con las pulsaciones regulares del metrónomo, 4, 2 y 1 respectivamente. Esto indica que la percepción auditiva concuerda con unidades sonoras exactas. A partir de la figura corchea los fraccionamientos no concuerdan con la unidad sonora exacta como se indica en la Figura 6.

En el contexto musical, se completan tiempos equivalentes a una pulsación. Por ejemplo, es necesario dos figuras corcheas para completar una figura negra, o cuántas semicorcheas son necesarias para completar una figura negra. 
Figura 6.

Parte de la unidad relativa y parte-parte

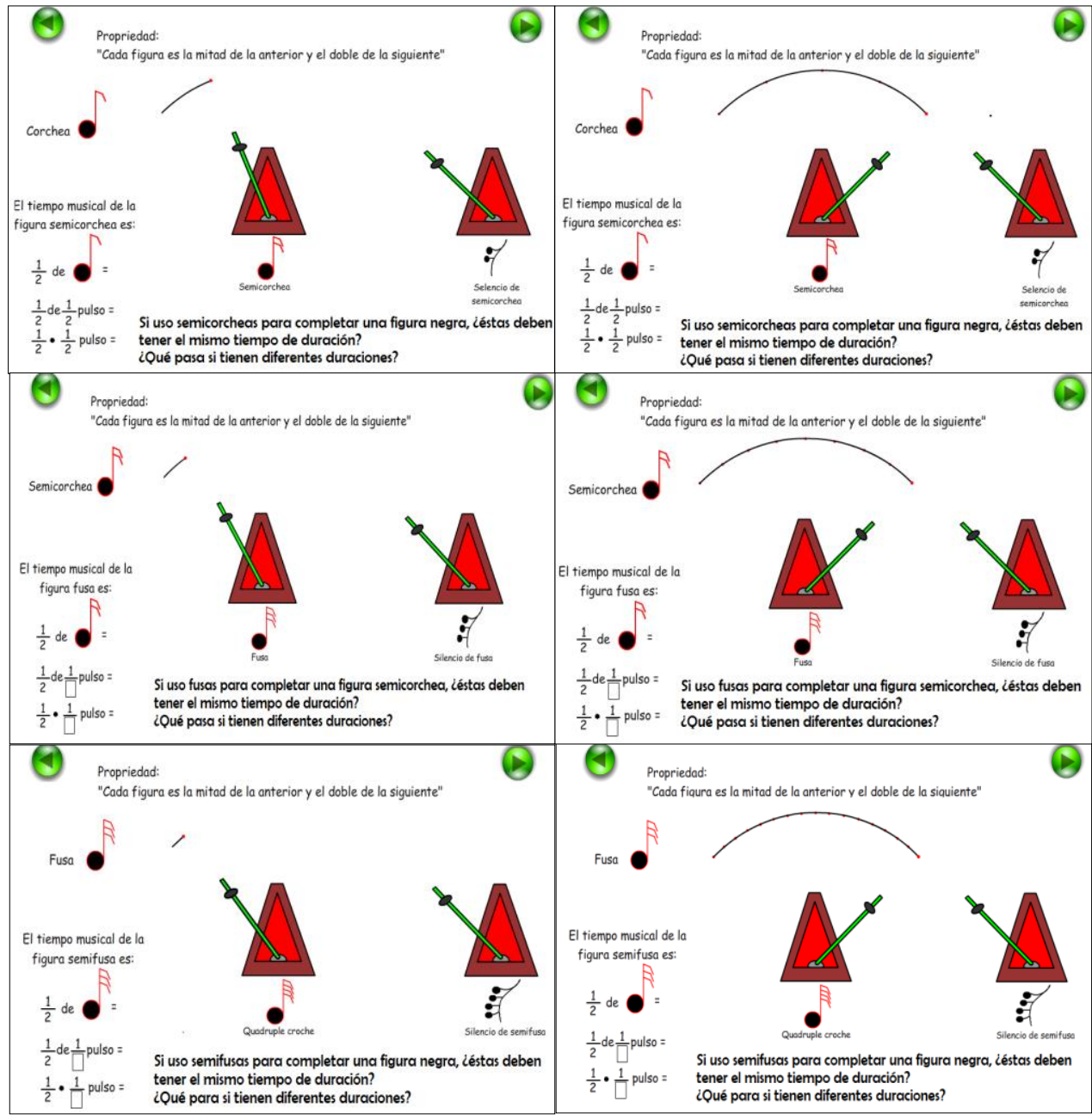

Fuente: Elaboración propia.

En esta situación podríamos decir que la unidad de referencia ha cambiado, una parte de la unidad inicial como es la figura negra, ahora la figura negra es una subunidad. Como refuerzo a la idea anterior planteamos preguntas como: ¿cuántas corcheas son necesarias para obtener una negra?, ¿cuántas fusas son necesarias para obtener una negra?, ¿cuántas negras se requieren para hallar una corchea? Estos cuestionamientos propician que el estudiantado construya equivalencias entre figuras para expresar una en términos de otras.

Alrededor de los cuestionamientos anteriores los profesores suelen manifestar una preocupación interesante: ¿cómo se trabajarían fracciones donde el numerador sea mayor que el denominador? Atendiendo dicha inquietud, en este contexto podríamos trabajar otro 
tipo de fracciones, por ejemplo es posible expresar $3 / 2$ con figuras blancas. Es decir, si sumamos tres veces la fracción de la duración de la figura blanca obtengo una fracción de duración de 3/2. Teniendo en cuenta la unidad con la que estamos operando, misma que tomaría dos connotaciones:

a. Si tomamos como unidad la figura redonda, 3/2 se expresa (bajo esta unidad) en un todo temporal $\left(\frac{3}{2} d e\right)$.

b. La figura blanca podría tomarse como unidad, entonces estamos identificando que la medida se refiere a la concepción de fracción como la suma de una fracción unidad, más que partes de un todo, de acuerdo con Olive y Vomvoridi (2006) y Tzur (1999). Esto quiere decir que $3 / 2$, es entendido como tres iteraciones de la figura unidad, en este caso 3 blancas. Aquí se puede percibir el papel que juega la unidad de medida como base fundamental para la construcción de espacio temporal musical.

\subsection{Equiparticiones}

Para Naik y Subramaniam (2008) en situaciones en las que el conteo se utiliza para llegar a la cardinalidad de un grupo concreto, el tamaño de cada unidad puede ser ignorado. Así, las niñas y los niños pasan por alto el tamaño de las partes; si se opera con números enteros consistiría en contar el número de partes sombreadas, y el número total de partes, como suele usarse en el modelo del pastel. En cambio, en contextos de medición, el tamaño de la unidad es indispensable.

Retomando el ejemplo de pastel, $\frac{3}{4}$ del pastel o de un rectángulo implícitamente sugiere la idea de equipartición, entonces: ¿el estudiante dará prioridad a esta propiedad?, ¿qué condiciones indican u obligan a que se tenga en cuenta que esta partición no es arbitraria?

Nuestra intención es aportar condiciones con las que el estudiante halle el significado y necesidad de aplicar una equipartición. En la métrica musical existen ciertas reglas temporales que se deben cumplir independientemente del intérprete y de lo contrario surgen consecuencias acústicas que nos señalan el cumplimiento o no de las reglas temporales. Si indagamos sobre las corcheas usadas para completar una negra, ¿deben tener igual tiempo de duración?, ¿qué pasaría si no tienen tiempos iguales? Estas preguntas abren la puerta para dos posibilidades que se representan en la Figura 7. 
Figura 7.

Representación de la equipartición

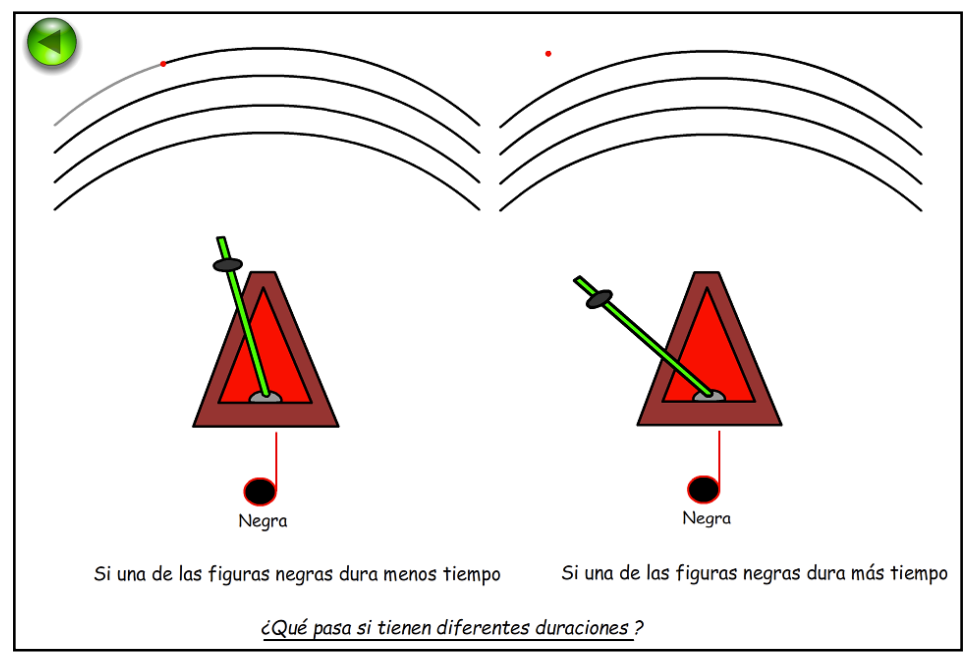

Fuente: Elaboración propia.

En la simulación se experimentan dos casos:

a. Cuando una de las figuras negras tiene menos tiempo de duración, esta representación le indica (traza, ubicación del punto rojo, tic-tac y sonido) al estudiante le faltaría un poco para completar el tiempo de duración de la figura redonda.

b. Cuando una de las figuras negras tiene mayor tiempo de duración, donde se percibe (traza, ubicación del punto rojo y sonido) que sobraría una parte para completar el tiempo de duración de la figura redonda.

En esta experimentación de tiempo musical induce a pensar al estudiantado que necesariamente las figuras negras representan equiparticiones de tiempo de la figura redonda y, en caso contrario, ocurre un fenómeno físico que no se ajusta a la métrica musical. La importancia radica en que existe una consecuencia acústica percibida por el estudiantado que le ayuda a dar un significado a las equiparticiones, situación que no se percibe en el ejemplo del pastel. Para afianzar el significado de equipartición, se puede extender a otras situaciones de equivalencias entre otras figuras y apoyarse en sus representaciones.

No deberíamos pasar por alto en este apartado que todo lo descrito en cuanto al pulso, hace referencia a composiciones o pasajes musicales en los que el pulso debe ser llevado de forma uniforme (por ejemplo: una marcha). Esta idea de uniformidad no podría generalizarse a toda la música; de hecho, existen multitud de expresiones musicales que 
permiten acelerar o decelerar el pulso (por ejemplo: rubatos, accelerandos, alargandos, etc.), independientemente de que las figuras musicales que aparecen en el pentagrama siguen siendo negras, redondas o cualquier otra.

\section{Algunas reflexiones}

Los artefactos computacionales usados en las dirección mencionada pueden hacer explícita la idea de unidad relativa, relación parte-parte y equipartición. Dichos artefactos abren otras posibilidades para que el estudiantado establezca una relación de orden entre las figuras y exprese una fracción en términos de otras determinadas por equivalencias. En este caso, no solo se establece una relación entre artefactos simbólicos (figura-fracción), sino que además tiene un significado de medida asociado al tiempo de duración de un sonido.

Naturalmente los objetos matemáticos carecen de medios de representación auditiva. Sin embargo, en estas actividades interdisciplinares queremos insinuar que, si articulamos artefactos simbólicos (los sistemas matemático y musical de signos) mediados por artefactos computacionales (representaciones dinámicas), podríamos lograr dar significado a un objeto matemático en el espacio musical. Es decir, si el estudiantado interactúa con la representación dinámica de la construcción de las figuras musicales asociadas a la estructura fraccionaria, de cierta manera cada estudiante está asignando un fenómeno acústico como forma de representación e identificación de una fracción.

La unidad que tomamos en este estudio, además de cumplir con las propiedades matemáticas de unidad, está dotada de características especiales por su naturaleza proveniente de un fenómeno sonoro. En esta contribución, nos referimos a "unidad" (en un principio al sonido de mayor tiempo de duración dentro del sistema de figuras musicales) y a partir de ella se construyen las demás figuras pertenecientes a dicho sistema.

La idea de unidad relativa que queremos introducir en este trabajo es una estructura susceptible a cambios en dos sentidos:

i) La variación del valor de su tiempo físico, que depende de cierta velocidad con que un fragmento musical es interpretado. Aunque el valor de su tiempo musical se conserva, la unidad puede durar 4.8 segundos, 3 segundos, 1.5 segundos, entre otros. Tiempos de referencia para deducir la duración de sus demás elementos.

ii) Con la aplicación a la unidad de la propiedad del sistema: que de cada figura es la mitad de la anterior y el doble de la siguiente, se generan fracciones de la unidad. 
Estas, en determinadas situaciones en el espacio musical, cambian unas por otras cumpliendo el papel de subunidades, por ejemplo, en los compases.

Desde nuestra perspectiva, pretendemos hacer una contribución al currículo desde el punto de vista interdisciplinar, ya que es importante que la población estudiantil encuentre en la matemática conexiones con otras disciplinas, reduciendo la idea generalizada de ver la matemática escolar como una disciplina aislada y lejos de su realidad. Asimismo, consideramos esta propuesta interdisciplinaria como un recurso didáctico que aporta al desarrollo de procesos cognitivos generados en actividades que involucran relaciones entre la noción de tiempo y las fracciones.

\section{Referencias}

Artigue, Michael. (2002). Learning mathematics in a CAS environment: The genesis of a Reflection about instrumentation and the Dialectics between Technical and Conceptual Work. International Journal of Computers for Mathematical Learning, 7(3), 245-274.

Conde, Alexander. (2009). Las fracciones al ritmo de la música. (Tesis para optar por el grado de Maestría en Matemática Educativa), Centro de Investigación y Estudios Avanzados del Instituto Politécnico Nacional, México D.F., México.

Conde, Alexander. (2013). Las unidad relativa como vínculo cognitivo entre el tiempo musical y las fracciones. (Tesis para optar por el grado de Doctorado en Matemática Educativa), Centro de Investigación y Estudios Avanzados del Instituto Politécnico Nacional, México D.F., México.

Conde, Alexander, Figueras, Olimpia, Pluvinage, François y Liern, Vicente. (2011). El sonido de las fracciones: una propuesta interdisciplinaria de enseñanza. Revista Suma, (68), 107-113.

Freudenthal, Hans. (1983). Fenomenología didáctica de las estructuras matemáticas (Trad. Luis Puig). México D.F.: Departamento de Matemática Educativa del CINVESTAV-IPN.

Galperin, Piotr y Georgiev, L.S. (1969). The formation of elementary mathematical notions. En Jeremy Kilpatrick y Izaak Wirszup (Eds.), Soviet studies in the psychology of learning and teaching mathematics (Vol. 1, pp. 189-216). Chicago, USA: The University of Chicago.

Gardner, Howard. (1997). Is musical intelligence special? In Verna Brummett (Ed.), Ithaca Conference '96: Music as Intelligence (pp. 1-12). New York, USA: Ithaca College Press.

Kieren, Thomas. (1976). On the mathematical, cognitive, and instructional foundations of rational numbers. In R. Lesch (Ed.), Number and measurement (pp. 101-149). Ohio: ERIC/SMEAC. 
Kieren, Thomas. (1983). La partición, la equivalencia y la construcción de ideas relacionadas con los números racionales. In M. Zweng, T. Green y J. Kilpatrick (Eds.), Proceedings of the Fourth International Congress on Mathematical Education (pp. 506-508). Estados Unidos: Birkhäuser.

Liern, Vicente (2011). Música y Matemáticas en educación primaria. Revista Suma, (66), 107-112.

Naik, Shweta y Subramaniam, K. (2008). Integrating the measure and quotient interpretation of fractions. International group of the psychology of mathematics education: Proceedings of the Joint Meeting of PME, 32, 17-24.

Olive, John y Vomvoridi, Eugenia. (2006). Making sense of instruction on fractions when a student lacks necessary fractional schemes: The case of Tim. Journal of Mathematical Behavior, 25(1), 18-45.

Parada, Sandra y Pluvinage, François. (2014). Reflexiones de profesores de matemáticas sobre aspectos relacionados con su pensamiento didáctico. Revista Latinoamericana de Investigación en Matemática Educativa, 17(1), 1- 31.

Parada, Sandra y Sacristán, Ana. (2010). Teachers' reflections on the use of instruments in their mathematics lessons: a case-study. International group of the psychology of mathematics education: Proceedings of the Joint Meeting of PME, 34, 25-32.

Piaget, Jean. (1978). El desarrollo de la noción de tiempo en el niño. México D.F.: Fondo de Cultura Económica.

Prediger, Susanne y Schink, Andrea. (2009). "Three eighths of which whole?" - Dealing with changing referent wholes as a key to the part-of-part-model for the multiplication of fractions. In M. Tzekaki, M. Kaldrimidou y C. Sakonidis (Eds.), International group of the psychology of mathematics education: Proceedings of the Joint Meeting of PME, 33, 409-416. Thessaloniki, Greece: PME.

Rauscher Frances, Shaw Gordon, Levine Linda, Wright, Eric, Dennis, Wendy y Newcomb, Robert. (1997). Music training causes long-term enhancement of preschool children's spatial-temporal reasoning. Neurological research, 19(1), 2-8.

Rauscher, Frances y Zupan, Mary. (2000). Classroom keyboard instruction improves kindergarten children's spatial-temporal performance: A field experiment. Early Childhood Research Quarterly, 15(2), 215-228.

Sánchez, María. (1999). Temporalidad, cronopsicología y diferencias individuales. Madrid: Centro de Estudios Ramón Areces.

Shilling, Wynne. (2002). Mathematics, music and movement: Exploring concepts and connections. Early Childhood Education Journal, 29(3), 179-184.

Steffe, Leslie, Cobb, Paul, y Glasersfeld, Ernst. (1988). Construction of Arithmetical Meanings and Strategies. New York: Springer-Verlag. 
Socas, Martín. (1997). Dificultades, obstáculos y errores en el aprendizaje de las Matemáticas en la Educación Secundaria. En Luis Rico, y otros (Eds), La Educación Matemática en la Enseñanza Secundaria (pp. 125-154). Barcelona: Horsori.

Still, Kathryn y Bobis, Janette. (2005). The integration of mathematics and music in the primary school classroom. Proceedings of Annual Conference of the Mathematics Education Research Group of Australasia. Building Connections: Theory, Research and Practice, 712-719. Sydney, Australia: MERGA.

Tiburcio, Susana. (2002). Música y matemáticas. Revista Elementos, Ciencia y Cultura, $8(44), 21-26$.

Trouche, Luc. (2005). An instrumental approach to mathematics learning in symbolic calculator environments. In The didactical challenge of symbolic calculators (pp. 137162). New York: Springer.

Tzur, Ron. (1999). An integrated study of children's construction of improper fractions and the teacher's role in promoting that learning. Journal for Research in Mathematics Education, 30(4), 390-416.

Vaughn, Kathryn. (2000). Music and mathematics: Modest support for the oft-claimed relationship. Journal of Aesthetic Education, (34), 149-166.

Vérillon, Pierre y Rabardel, Pierre. (1995). Cognitions and artifacts: a contribution to the study ofthought in relation to instrument activity. European Journal of Psychology of Education, 10(1), 77-101.

Vygotsky, Lev. (1988). El desarrollo de los procesos psicológicos superiores. México D.F.: Grijalbo. 